\title{
Federalismos unitarios: constitución paradigmática y nuevos federalismos ${ }^{*}$
}

\section{Unitary Federalisms: paradigmatic constitution and new federalisms}

Fecha de recepción: 15 de septiembre de 2011

Fecha de evaluación: 7 de octubre de 2011

Fecha de aprobación: 10 de octubre de 2011

LIIIANA Estupiñán ACHURY**

\section{RESUMEN}

El artículo reflexiona sobre la distancia que toman en el plano real diversos Estados federales con relación a la constitución territorial paradigmática o constitución norteamericana de 1787; tramo que ha dado lugar a la construcción de diversos federalismos, incluso algunos de ellos más a tono con lógicas centralistas que con los principios de soberanía y autonomía.

Palabras clave: constitución paradigmática, constitución derivada, federalismo, centralismo, autonomía territorial, competencias.

Resultado del proyecto de investigación terminado en 2010: "La constante estructural centralista de la forma de Estado colombiana". Proyecto adscrito a la línea de investigación en: Territorio, descentralización y democracia del grupo de Derecho Público de la Facultad de Jurisprudencia de la Universidad del Rosario.

** Doctora en Sociología Jurídica e Instituciones Políticas de la Universidad Externado de Colombia (2010). Abogada, especialista en derecho constitucional, derecho administrativo y magíster de la Universidad Libre. Profesora de carrera de la Facultad de Jurisprudencia y directora de la línea de investigación en: Territorio, descentralización y democracia del grupo de Derecho Público A1 de la Facultad de Jurisprudencia de la Universidad del Rosario. Correo electrónico: liliana.estupinan@urosario.edu.co. Dirección correspondencia: Calle 44 D No. 45 - 45, interior 6, apartamento 904 . 


\section{ABSTRACT}

This article analyzes the distance several federal states take in the real plane in regard to the paradigmatic or territorial constitution or U.S. Constitution of 1787; This stretch has led to the construction of various federalisms, with even some of them more in tune with centralist logics than with the principles of sovereignty and autonomy.

Keywords: Paradigmatic constitution, derived constitution, federalism, centralism, territorial autonomy and competence.

\section{INTRODUCCIÓN}

El concepto de federalismo aparece hoy como una categoría un tanto difusa, al menos en lo que se refiere a reconocer con claridad sus rasgos diferenciables con el regionalismo (o el sistema autonómico). La pluralidad de nominaciones que se añaden con frecuencia a las categorías tradicionales (dual o cooperative federalism) no hace sino confirmar las dificultades para una correcta delimitación conceptual (creative federalism, extensive federalism, new federalism).

\begin{abstract}
Es más probable que la tendencia más marcada contemporánea sea aquella que apunta hacia la "hibridación" o "clonación" de los modelos. Por ello sospechamos que empieza a no tener sentido el esfuerzo de la dogmática por simplificar bajos estrictos standard teóricos, realidades político-constitucionales que comparten, al final, su adscripción a ese fenómeno generalizado, al que Häberle ha bautizado como "regionalismo" y del que se dice es un "principio estructural naciente del estado constitucional (RuizRico, 2001: 25-26).
\end{abstract}

El primer objetivo específico de la investigación indagó sobre las variadas tendencias a las que están sometidos los modelos territoriales y sus niveles intermedios de gobierno, llámense departamentos, estados, länder, cantones, comunidades autónomas, regiones o provincias, según sea la expresión que se utilice en los textos constitucionales.

Para ello, se hizo un acercamiento a diversos modelos territoriales, en su mayoría en proceso de afianzamiento de la descentralización, e incluso algunos de ellos muy a tono con la tendencia federal mundial y, por supuesto, inspirados o condicionados por los retos que impone la globalización a los Estados nacionales y sus correspondientes divisiones político-administrativas.

Tres enfoques se presentan a la hora de estudiar los modelos territoriales o las formas de Estado. Al final, una combinación de todos ellos proporcionó el marco teórico y metodológico necesario para comprender el modelo territorial colombiano y el papel de su nivel intermedio de gobierno. El primero, identificado con la "teoría de los tipos ideales de las formas de Estado". Un enfoque tildado de "tradicional", en tanto describe y sistematiza los rasgos característicos de las diversas formas de Estado, denominadas Estado unitario, Estado regional y Estado federal. Por supuesto, una propuesta que se encuentra limitada y en crisis ante las innumerables versiones dadas sobre la distribución territorial del poder en el plano real, a tal extremo que la doctrina señala una cuarta forma de Estado: la devolution. De hecho, se observan múltiples formas de Estados unitarios, de Estados regionales e incluso diversos modelos de federalismo. En cada uno de ellos el nivel intermedio cumple un papel disímil e incluso sui generis para la doctrina tradicional de las formas de Estado.

El segundo enfoque reflexiona sobre el papel de las constituciones modelo o paradigmáticas en el tema territorial. Estas han sido imitadas por imperio o por prestigio. Por lo tanto, se trata de 
una propuesta que compara el modelo territorial por estudiar con la constitución paradigmática que lo inspiró. Con ello, se permite establecer las similitudes o diferencias entre una constitución específica y la constitución paradigmática. Así, he identificado como constituciones territoriales paradigmáticas la norteamericana, la española y la francesa.

El tercero, estudia las constituciones territoriales a partir de su cercanía o distancia de los principios extremos que recogen el tema de la distribución territorial del poder, esto es, centralización y descentralización. Poco importa la forma de Estado literalmente consignada en el texto constitucional, en tanto el desarrollo real de los principios territoriales, independientemente del nombre adoptado, permita encontrar constituciones federales con altos niveles de centralización, o Estados unitarios con grados considerables de descentralización.

En este contexto, el título de federalismo unitario reflexiona, tal como se ha señalado en el resumen, sobre la distancia que toman en el plano real diversos Estados federales con relación a la constitución territorial paradigmática o constitución norteamericana de 1787 . Hoy se habla de diversos federalismos, varios de ellos más a tono con lógicas centralistas que con los principios de soberanía y autonomía.

\section{LA CONSTITUCIÓN FEDERAL PARADIGMÁTICA}

La Constitución de Filadelfia de 1787 es un hito para la teoría del Estado, en tanto que creó la forma de gobierno presidencial y acomodó la fortaleza de sus partes en un todo denominado "federalismo", una fórmula mágica que le ha permitido a Estados Unidos subsistir en medio de las diferencias, en no menos de doscientos años. Un pacto que "lejos de significar la abolición de los gobiernos de los estados miembros, los convirtió en partes constituyentes de la soberanía nacional" (Hamilton,
Madison y Jay, 1941: 35) ¿Pero cuál fue la fórmula del pacto? ¿Por qué se habla hoy de varias clases de federalismo e incluso del federalismo unitario?

El pacto se caracterizó por la construcción de una constitución escrita que recreó la teoría de la división del poder, cifrada en un legislativo, un ejecutivo y un poder judicial, bajo el respeto de las estructuras de poder ya preexistentes en las partes. Una constitución estratégica para efectos de la paz, la guerra, el comercio, asuntos debidamente tasados y limitados en beneficio de las exorbitantes competencias de sus divisiones subnacionales. Así, todo aquello no enumerado en el texto político a manera de cláusula residual se concibió en beneficio de las partes. Igualmente, un orden constitucional que concibió la existencia de una cámara legislativa integrada por representantes de los Estados y un tribunal constitucional para la resolución de conflictos, "titular del derecho de declarar ultra vires las decisiones que no respeten los principios de separación y de autonomía" (Croisat, 1995: 32).

El éxito del modelo se cifra en la fórmula que lo vio nacer, esto es, un pacto entre iguales competentes tanto para crear la constitución como también para reformarla. Sin duda, una fórmula audaz, pero que no por ello dejó de despertar inquietudes y recelos. Por ello, Alexander Hamilton, en el texto clásico El federalista, respondió una y otra vez a la necesidad imperiosa de organizarse como un Estado federal y superar la etapa confederal (17781787), dado que bajo este esquema preveía futuras divisiones, celos e inequidades que darían al traste con la construcción del Estado norteamericano. Decía Alexander Hamilton (1941): “Es necesario que un hombre se halle muy absorto en especulaciones utópicas para poner en duda que si los Estados estuvieran completamente separados o sólo unidos en confederaciones parciales, las subdivisiones en que podrían partirse, contenderían frecuente y violentamente unas con otras" (19). 
Por ello, el federalismo norteamericano se origina a partir de la unión de Estados independientes y soberanos, proceso denominado por la doctrina "bottom-up process", (Lothar, 2006: 16), en contravía de la construcción de federalismos a partir de la fragmentación de un Estado unitario, denominado "top-down". En el origen del pacto bien puede cifrarse como parte del éxito de la constitución modelo; en otras palabras, para CoIombia el federalismo representó, a comienzos del siglo XIX, vientos de reconquista y sentimientos de vulneración; para los angloamericanos, dicha opción significó el logro de la independencia y la fundación de un imperio.

A partir del federalismo norteamericano se construyen las diversas denominaciones de federalismo. Del federalismo dual, como se ha llamado al modelo territorial original de los Estados Unidos -caracterizado por una plena y clara división entre las tareas desarrolladas por el Estado federal y los estados federados- se pasó al denominado federalismo de cooperación, cuyo rasgo es la "colaboración" entre el nivel central y el subnacional; un rasgo que al degenerarse implica la considerable injerencia del centro en los asuntos de la periferia, o un bloqueo de la periferia frente a las tareas del centro.

Sobre esta transformación, Gerardo Ruiz-Rico Rico (2001) anota:

El tránsito del dual federalism al cooperative federalism ha sido posible gracias a construcciones jurisprudenciales como la elaborada por el Tribunal Supremo norteamericano a través de su doctrina de los implied powers. En ella se resume la necesidad de ampliar la esfera de competencias del poder central, más allá de la tasada enumeración de potestades que le atribuye la Constitución Federal, y la existencia en consecuencia de amplios ámbitos concurrenciales entre federación y estados. De hecho, sin esa concepción flexible y abierta de la estructura de división de competencias formalizada en la norma fundamental difícilmente el modelo federal hubiera podido hacer frente a la asunción de los compromisos sociales de un new deal (21).

El federalismo de cooperación también se caracteriza porque el Estado generalmente dicta las normas base o marco, quedando los estados miembros con la atribución de adaptarlas e incluso ejecutarlas. Si el estado miembro se dedica meramente a la ejecución, el federalismo asume la alocución de "federalismo de ejecución".

En materia de reparto competencial, surgen diferencias entre los sistemas; así, para el caso norteamericano, la distribución se caracteriza por una relación restringida, limitada y basada en el texto constitucional de competencias radicadas en la federación y una cláusula residual en favor de los estados federales o divisiones subnacionales. Significa esto que a la federación le corresponde garantizar la seguridad, la paz y la pervivencia de la unión, en beneficio de las vastas competencias de las partes miembros. Por su parte, para el federalismo de cooperación es común la relación de sendas listas de distribución competencial, esto es, competencias exclusivas tanto de la Federación como de los Estados miembros y sendos listados de competencias concurrentes.

En el tema jurisdiccional también se suscitan diferencias. Por ejemplo, para el federalismo cooperativo alemán dicha potestad es básicamente materia de los Länder: "sólo hay cinco tribunales federales y el Tribunal Constitucional Federal como el Ilamado "guardián de la Constitución" (Häberle, 2006: 14). Sin embargo, el federalismo europeo se caracteriza, generalmente, por la existencia de una sola jerarquía de tribunales, contrariamente a la organización de la justicia en los Estados Unidos (López, 1994: 87).

El federalismo también se caracteriza por la existencia de dos cámaras. Para el caso norteamericano -federalismo dual-, la participación de los estados está dada en la Cámara Federal o Senado, 
en donde estos poseen el mismo derecho de voto, con independencia de su tamaño. Los representantes estatales son elegidos directamente por el pueblo conforme al texto de la enmienda XVII de 1913 y forman parte del poder legislativo (Lothar, 2006: 23). En Alemania -a manera de ejemplo de federalismo de cooperación-, existen al igual que en Estados Unidos dos cámaras, el Bundestag, la cual representa los intereses federales, y el Bundesrat, donde participan los gobiernos de cada uno de los dieciséis estados miembros o Länder. A diferencia del modelo norteamericano, la ponderación de votos se da según el tamaño de los estados miembros o Länder. En cuanto a la designación, en tanto para los norteamericanos procede la elección directa, para los alemanes la designación la realiza el gobierno de la división subnacional, "es decir, representantes del ejecutivo, de modo que los votos de un Land únicamente pueden ser emitidos en bloque o sometidos a indicaciones del ejecutivo del Land" (Lothar, 2006: 23).

Paloma Biglino Campos (2007: 105 y ss.) explica las diferencias entre el federalismo norteamericano $y$ las diversas versiones de federalismo europeo, a partir de la irrupción tardía de dicho modelo en el viejo continente, de forma contraria a la extraordinaria influencia del pensamiento político francés, caracterizada por el centralismo, además de la imagen poco afortunada que tuvo la guerra de secesión norteamericana (1861-1865) en Europa y a la identificación que tuvo el federalismo para los dirigentes políticos del siglo XIX, con las teorías de la soberanía popular. A pesar de ello, Europa construye el Estado liberal, eso sí, sin desprenderse del pasado, una mixtura entre monarquía, nobleza y principios liberales, en donde el federalismo asumió un peso en la historia, ligado a la reivindicación de la diversidad, la pluralidad y los nacionalismos.

Por ello, se encuentran diversas versiones de federalismo en Europa. Tres de ellos son paradigmáticos a la hora de entender las innumerables posibilidades de construcción federal. Señala Peter Häberle:

\begin{abstract}
Los modelos se mezclan y cambian, de ahí que utilice el término "metamorfosis" del federalismo. Así por ejemplo, al comparar los tres países de habla germana, Suiza sería el país con un federalismo más fuerte, Austria la de carácter más unitario y Alemania se encontraría en la mitad del camino. [...] En resumen, el Estado federal depende de cómo se pueda en el tiempo y en el espacio lograr un balance entre la imprescindible homogeneidad (unidad) y la pluralidad óptima (diferencia y pluralidad) (2007: 178).
\end{abstract}

\section{LA CONSTITUCIÓN FEDERAL DERIVADA}

Un mero acercamiento a la historia del federalismo alemán permite comprender las diversas expresiones que se le endilgan a esta forma de Estado; igualmente, desmitifica la afirmación que señala que los máximos niveles de descentralización, por lo menos en teoría, corresponden a la forma de Estado federal.

En general, se señala como punto de partida, para el modelo federal alemán, la Federación de Alemania del Norte de 1867, cuya forma territorial se reafirmó por las constituciones de Bismarck (1871) y de Weimar (1919). Por supuesto, sin desconocer la preexistencia material de estructuras federales, como la Federación Alemana de 1815/1820 y en la Edad Media, la existencia de muchos principados, ciudades y obispados (Häberle, 2006: 13). Pero sin duda, fue la Constitución de 1949 (Arroyo, 2006: 26) la que consagró en forma definitiva el Estado federal alemán, hecho consolidado con la unificación propiciada en 1990.

Sobre la construcción federal alemana, Fernando Domínguez García (2005) apunta que dicho modelo

nace de un proceso de integración que sigue los parámetros federales, excepto en el paréntesis nazi. Es muy difícil concretar el momento en que se puede comenzar a calificar a Alemania como un Estado compuesto, puesto que depende de qué calificación jurídica se otorgue a 
la Confederación de Alemania del Norte, aunque es pacífico que el Segundo Reich era una estructura federal donde los Estados conservaban gran parte de su autonomía. A partir de ese momento se puede realizar una comparación del grado de autonomía política de los futuros Länder, que partiría de una posición fuerte, para pasar en la República de Weimar a una situación mucho más lánguida, y luego volver a un escenario más confortable en el seno de la República Federal de Alemania (aunque sin los privilegios ni las atribuciones del Segundo Reich), que se ha tenido que ir modificando a causa del proceso de integración europea (140).

Para la década de los años sesenta del siglo XX surge una frase que representa el nivel de desarrollo del federalismo alemán. Se lee: "El Estado federal alemán contemporáneo es, en principio, salvo ciertas limitaciones, 'un Estado federal unitario', una idea que simboliza el predominio del centro frente a la mermada actuación de los niveles subnacionales" (Hesse, 2006: 438). Un calificativo adjudicado a partir del desbalance dado entre los poderes de la Federación (Bund) y el limitado papel de los estados o Länder (439); ello en razón de la considerable pérdida de competencias y autonomía, resultado del desplazamiento de potestades legislativas a la Federación, y más tarde a las comunidades europeas (Von Danwitz, 2002: 26).

"La unitarización del Estado federal alemán" ha sido un hecho aceptado durante varias décadas, conforme lo señala el doctrinante Von Danwitz, con tanto silencio e indiferencia como firmeza (16). Tendencia centralista caracterizada por la merma de las facultades soberanas de los Länder desde la época de la posguerra, durante los años ochenta e incluso durante el proceso de reunificación, un rasgo distintivo de la República Federal, potenciado por las reformas constitucionales, la interpretación del Tribunal Constitucional Federal, e incluso por la propia actitud de los Länder (Arroyo, 2006).
El federalismo cooperativo propio del proceso de reunificación no se separó de los rasgos unitarios característicos del federalismo alemán, en parte, porque durante esta época primaron los procesos de autocoordinación y de equiparación del nivel de vida de los Länder, en un difundido afán de homogeneidad como expresión de igualdad y solidaridad (Von Danwitz, 2002: 26). Häberle (2007) titula esta etapa con el nombre de "federalismo de garantía", caracterizado por la solidaridad y la gigantesca ayuda financiera de la Federación y los Estados occidentales a los cinco nuevos Länder; un pacto que supone la transferencia cada año de 150.000 millones de marcos, esto es, un paquete de solidaridad proyectado hasta el año 2019 (177).

Se hace pertinente, entonces, la expresión de "federalismo de ejecución", la cual hace alusión al considerable papel del Bund, muy a pesar de la competencia general y residual a favor de los Länder, que se caracterizan más por la ejecución de la mayor parte de la normativa federal que por el ejercicio de la potestad legislativa. Así, el federalismo unitario alemán se caracteriza por los limitados poderes legislativos del Land (Domínguez, 2005: 138). Potestad evidentemente deteriorada y agravada bajo la incidencia del derecho comunitario. A manera de ejemplo, señala Thomas Von Danwitz (2002) que "la cantidad de textos sobre asuntos de las Comunidades Europeas que se presentan al Bundesrat superan muy considerablemente los proyectos de leyes nacionales que llegan a sus manos, e incluso son superiores al número de decretos jurídicos sobre los que también debe decidir el Bundesrat" (20). En el mismo sentido, Hans-Peter Schneider (2009: 11-37) anota que muchas de las competencias legislativas que correspondían a los parlamentos de los Länder emigraron directamente hacia Bruselas, perdiendo así la competencia el nivel federal; a manera de ejemplo, el caso de la televisión "en donde la Comisión Europea reclamó su propia competencia en el marco de la regulación de la libre circulación de servicios en el mercado interior" (15). 
Superar el federalismo cooperativo y de ejecución en la búsqueda de un "federalismo de competencia"1 (Kluth, 2007: 53) fueron las banderas que precedieron la última reforma de la Ley Fundamental de Bonn, esto es, una clara separación de los ámbitos competenciales, hecho que se concreta en una amplia diferenciación de las facultades legislativas y una reforma a la Constitución financiera federal o, como lo señala Klaus-Júrgen Nagel (2002: 65-99), la consecución de un "federalismo asimétrico" que fortalezca el rol legislativo de los Länder, la limitación a la legislación concurrente, la especificación del derecho federal por promulgar, la supresión de tareas conjuntas, la reducción de la financiación compartida y el fortalecimiento de la autonomía fiscal (66).

El acuerdo final del paquete de reformas anunciado el 14 de diciembre de 2005 mediante una declaración conjunta de la canciller y de los jefes de gobierno de los Länder anunciaba la consecución de las siguientes metas:

1. Fortalecimiento de la legislación de Bundy Länder a través de una delimitación más clara de las competencias y la eliminación de la competencia marco.

2. Desactivación de los bloqueos recíprocos a través de la reelaboración de la necesidad de consentimiento de las leyes federales en el Bundesrat.

3. Desmantelamiento de la financiación mixta y nueva redacción de las posibilidades de financiación a través de ayudas económicas del Bund previstas en el Pacto de Solidaridad II para los nuevos Länder.

$\overline{1}$ Winfried Kluth señala que la reforma estaba orientada en el plano del modelo del federalismo competitivo, "sin que ello signifique, sin embargo, que los elementos solidarios desaparezcan por completo. Esto se demuestra sobre todo en el hecho de que los supuestos de financiación mixta no hayan sido totalmente suprimidos y también en que la reforma de la Constitución financiera, incluido el equilibrio financiero de los Länder, haya quedado excluida" (Kluth, 2007: 53).
4. El fortalecimiento de la idoneidad europea de la Ley Fundamental (Kluth, 2007: 53).

Así, en el año 2006 se aprueba la mayor reforma a la Ley Fundamental de Bonn, acompañada por las demás leyes que se relacionan con la revisión constitucional o ley de acompañamiento a la reforma del federalismo ( Föderalismusreform-Begleitgesetz); una revisión que introdujo cuarenta modificaciones al texto de la Ley Fundamental.

Hans-Peter Schneider pondera el esfuerzo político que condujo a la transformación parcial del modelo territorial alemán; muy a pesar de los interrogantes y vacíos que se objetan, su afirmación se realiza en razón de los reiterados fracasos de los intentos de reforma que se impulsan en diferentes Estados federales. En concreto, Austria, cuya gran Convención concluyó que no había nada que hacer en este ámbito; igualmente, los canadienses con más de dieciséis años desde el fracaso del acuerdo de Charlottetown (Consensus Report on the Constitution); asimismo, Australia que fracasó en el intento de reformar la estructura provincial, que pretendía reforzar estas entidades frente a los municipios, y por último, el caso de Pakistán, en su frustrado esfuerzo de construcción de una autonomía local (2009: 13).

El objeto de la reforma, siguiendo a María Ángeles Martín Vida (2006: 161-194), fue, por un lado, reducir el número de Zustimmungsgesetze, esto es, leyes federales que requerían de doble aprobación, por parte del Bundestag y del Bundesrat; este último, denominado por la doctrina contraparlamento $^{2}(163)$, en tanto bloqueaba el procedimiento legislativo, además de los constantes enfrentamientos políticos entre Estados y Federación. En otras palabras, una forma de reducir la influencia de los Länder en el procedimiento legislativo federal.

2 Expresión de B. Ulrich (ibídem: 163). 
El otro aspecto de la reforma se dio con la necesidad de contrarrestar el centralismo legislativo; para ello, disminuyó, a favor de los Länder, las competencias legislativas de la Federación. Esto, conforme lo anota María Ángeles Martín Vida, se realizó por varias vías:

Ampliación de la competencia legislativa exclusiva de los Länder, supresión de la legislación marco y reordenación del catálogo de materias sujetas a legislación concurrente, con una reducción del ámbito de aplicación de la "cláusula de imprescindibilidad"3 (164), del artículo $72.2 \mathrm{GG}$, pero a la vez con introducción de la posibilidad de que los Länder puedan en numerosos ámbitos apartarse, mediante normas con rango de ley, de la legislación federal (2006: 162-163).

También tuvo como objeto la disminución de los supuestos de financiación mixta en el ámbito de las tareas comunes (Kluth, 2007: 55-56). Se trata de una reducción significativa "sometida a largos plazos transitorios en lugar de una completa supresión, tal y como en un principio se demandaba" (Martín Vida, 2006: 56). En general, los Länder deben contribuir con una parte significativa en la financiación de las tareas comunes ${ }^{4}$.

Ahora bien, sobre el alcance de la reforma en asuntos de regulación europea, se limitó la representación de la Federación a su ejercicio por un ministro de Land, con el fin de dar mayor participación a los Estados en las sesiones del Consejo; además, se introdujeron "nuevas regulaciones en virtud de las cuales los Länder han de participar de las multas pecuniarias que la Federación haya

3 Debates suscitados por el ejercicio legislativo que realizaba la federación en materias objeto de competencia legislativa concurrente, excluyendo con ello la posibilidad de que los Länder legislen por ellos mismos, aduciendo que dicha legislación federal era imprescindible para establecer o garantizar "relaciones vitales equivalentes o asegurar la unidad económica y jurídica del Estado alemán" (Martín Vida, 2006: 164).

4 Mejora de la estructura económica regional, de las estructuras agrarias y de la protección de las costas; fomento de la investigación al margen de los centros de enseñanza superior y edificación de centros de enseñanza superior. de pagar a la Unión Europea como consecuencia de una transposición tardía de las directivas o a causa de las lesiones de las disposiciones sobre el déficit presupuestario" (Martín Vida, 2006: 56-57).

Adicionalmente, se dictaron algunas medidas para proteger a los municipios, entre otras, la prohibición de traslado directo de competencias entre la federación y el ente local (ley federal); esto implica que dicho traslado sólo puede darse de la federación al Land, que decide la conveniencia o no de trasladar la tarea al ente municipal. Por otra parte, se fijó expresamente la capitalidad de Berlín, "además, que la representación del Estado global en la capital sea tarea de la Federación" (Martín Vida, 2006: 57).

Varios interrogantes se suscitaron de forma inmediata a la aprobación de la reforma territorial; los primeros sobre el impacto o problemas que se esperarían en el desarrollo de la nueva normativa, y los segundos relacionados con los vacíos de la revisión que llevarían a la necesaria creación de una nueva agenda de trabajo.

Sobre el impacto de la normativa, por ejemplo, y desde el punto de vista técnico-jurídico, se plantearon dudas sobre el ejercicio del derecho que tendrían los Länder de apartarse de la legislación federal (Martín, 2006: 191); de la misma forma, la incertidumbre y dificultad que tendrían los operadores jurídicos para identificar las normas aplicables e incluso las dificultades que podrían tener los órganos de la Unión Europea para saber si una directiva ha sido correctamente implementada en el Estado alemán.

Sobre los asuntos que quedaron pendientes, señala Martín Vida (191): (i) que algunos sectores plantearon la evidente necesidad de reestructurar la organización territorial a través de la fusión de algunos Länder pobres o pequeños 5 . Sobre este punto,

5 Häberle es crítico respecto de esta propuesta, para lo cual anota: "El Ministro Federal de Hacienda H. Eichel osó, incluso, con 
Peter Häberle (2007) señala que de ninguna forma se deben fusionar estados por asuntos financieros o económicos, en tanto estos deben subsistir por asuntos de pluralismo y singularidad (182). (ii) Otro asunto es el relacionado con la economía presupuestaria o la presencia de crisis presupuestarias; para ello, Winfried Kluth (2007) propone establecer un sistema de alerta temprana de reconocimiento y lucha frente a las crisis, desarrollo de criterios de endeudamiento aceptable, establecimiento de una serie de instrumentos que hagan posible la ejecución de dichos criterios, diferenciaciones estructurales entre los Länder y asientos de datos comparables, además de conceptos de saneamiento y autonomía ampliada (p. 60). (iii) Además, sobre el tema de la superación del desequilibrio territorial o la distancia económica entre unos Ländery otros, punto susceptible de intervención sólo hasta la expiración del Pacto de Solidaridad II en el año 2019; y finalmente (iv) el de la necesaria democratización del Bundesrat o elección directa de sus miembros (Schneider, 2009: 27).

Al final, y tal como lo anotan Michael Lothar $y$ Winfried Kluth, se construyó un federalismo experimental que conserva los componentes del Estado unitario, del federalismo cooperativo e incluso del federalismo solidario, meramente atenuados con la última reforma.

A pesar de las críticas y los temores anotados frente a las falencias o al impacto negativo o positivo de la reforma estudiada, es innegable que el federalismo en Europa es una tendencia en materia de distribución de poder y para el caso alemán es sinónimo de libertad. Uno de sus teóricos más decantados, Peter Häberle, supera el análisis meramente competencial a partir de una mirada cultural y de altruista solidaridad entre los estados

argumentos fiscales y económicos, poner en cuestión la existencia de los Länder federales 'pobres' de Bremen (sobre ello, Frankfurter Allgemeine Zeitung del 20 de abril de 2004, p. 12: "Bremen se hunde en deudas") y de Saarland, y osó reiterar con ello un viejo debate sobre la reestructuración del territorio federal y la creación de Länder 'más fuertes'" (Haberle, 2006: 16). miembros. Así, el doctrinante comprende que el federalismo es un proceso inacabado y de reconstrucción permanente; su frase "la libertad alemana es libertad federal" conduce a la idea de no dar un paso atrás en materia de descentralización, pero eso sí, entendiendo día a día las dificultades y los diferentes matices que toman el camino hacia la autonomía y el respeto a la diversidad cultural.

"Un Estado unitario sería hoy, como ya hace mucho tiempo, inimaginable y, como en el 'Tercer Reich' y en la República Democrática Alemana, una catástrofe con pérdida total de libertad" (Häberle, 2006: 12-13), frase que presenta un traspié al leer las cifras que trae Hans-Peter Schneider sobre la percepción ciudadana acerca del Land y del federalismo en Alemania. Según la encuesta, la cuarta parte de los ciudadanos alemanes considera a los Länder prescindibles; sólo el $3 \%$ de los ciudadanos de los Länder piensa en la política del Land; los ciudadanos se identifican primero con su ciudad o pueblo y luego con el plano federal o comunitario, y en más de la mitad de los Länder este ente territorial se mencionó en último lugar. Finalmente, el $91 \%$ de los encuestados son partidarios de estándares comparables a nivel federal en materia de jardines de infancia, escuelas y universidades, y el $85 \%$ de que las tarifas impositivas se fijen a nivel federal (Schneider, 2009: 34). Después de la valoración ciudadana, concluye Hans-Peter Schneider con la siguiente frase: "Alemania: $i$ un Estado federal sin federalistas?" (33-34). Una respuesta que conlleva a pensar en la existencia de dos imaginarios territoriales: para el caso federal, uno es el imaginario de la ciudadanía, y el otro, el de los políticos y el de los académicos. En general, un sistema también objeto de controversia e inconformidad, a pesar de representar "teóricamente" el máximo nivel de libertad, autodeterminación y autonomía.

\section{CONCLUSIÓN}

La teoría de las formas tradicionales de Estado, esto es: Estado unitario, federal y regional, hoy se 
encuentra plenamente desdibujada y en evidente cambio y transformación. Ningún Estado es completamente centralizado; sin duda, la descentralización es la tendencia en materia de la relación entre territorio y poder, así como el federalismo es la moda en asuntos de modelos territoriales. Por ello, se encuentran Estados unitarios con indudables procesos de descentralización o Estados regionales que se confunden en la práctica con modelos federales, e incluso modelos federales tildados de unitarios. Sin duda, el caso alemán, tildado de federalismo unitario, sirvió para comparar dicho modelo con la Constitución paradigmática norteamericana de 1787, no por ello exenta de ajustes y contradicciones, o los cuestionamientos que se hacen en torno al carácter centralista de los federalismos latinoamericanos.

\section{REFERENCIAS}

Arroyo, A. (2006). El federalismo alemán en la encrucijada. Sobre el intento de modernización del orden federativo en la República Federal de Alemania. Madrid: Centro de Estudios Políticos y Constitucionales.

Biglino, P. (2007). Federalismo de integración y de devolución: El debate sobre la competencia. Madrid: Centro de Estudios Políticos y Constitucionales.

Croisat, M. (1995). El federalismo en las democracias contemporáneas. Barcelona: Hacer.

Cueto, C. (2001). La organización de Estado austríaco: ¿un caso de regionalismo o federalismo unitario? Revista de Estudios Regionales.

Domínguez, F. (2005). Las regiones con competencia legislativa. Un estudio comparado de su posición constitucional en sus respectivos Estados y en la Unión Europea. Valencia: Tirand Lo Blanch.

Häberle, P. (2006, mayo-agosto). El federalismo y el regionalismo: una estructura del Estado constitucional. Experiencias alemanas y proyectos.
Memorándum para un proyecto español. Revista Española de Derecho Constitucional, 77.

Häberle, P. (2007, julio-diciembre). Comparación constitucional y cultura de los modelos federales. ReDCE, 8.

Hamilton, A., Madison, J. y Jay, J. (1941). El federalista. Los ochenta y cinco ensayos que Hamilton, Madison y Jay escribieron en apoyo de la Constitución norteamericana. México, D.F.: Fondo de Cultura Económica.

Hesse, K. (2006, julio-diciembre). El Estado federal unitario. ReDCE, 6.

Kluth, W. (2007). La reforma del federalismo alemán: razones, objetivos y modificaciones. $R e$ vista de Derecho Político, 70.

López, L. (1994). Introducción al derecho constitucional. Valencia: Tirand Lo Blanch.

Lothar, M. (2006, julio-diciembre). El Estado federal Experimentado. ReDCE, 6.

Martin, M. (2006, julio- diciembre). La reforma del federalismo alemán, cambios en el reparto material de competencias entre la federación y los Länder. ReDCE, 6.

Nagel, K.J. (2002). El federalismo alemán. ¿Más cooperación o nueva asimetría? Revista de Estudios Políticos, 118.

Ruiz-Rico, G. (2001). Los límites constitucionales del Estado autonómico. Madrid: Centro de Estudios Políticos y Constitucionales.

Shneider, H.P. (2009). La reforma del federalismo en Alemania. Fines, negociaciones, resultados. Revista d'Estudis Autonomics i Federals, 8.

Von Danwitz, T. (2002). La colaboración en la estructura de Estado. La experiencia alemana. Revista de Estudios Autonómicos, 1. 